Strahlenther Onkol 2013 · 189:803-803

DOI 10.1007/s00066-013-0421-3

Online publiziert: 31. Juli 2013

c) Springer-Verlag Berlin Heidelberg 2013

J. Schultze

Klinik für Strahlentherapie, Universitätsklinikum Schleswig-Holstein (UKSH), Campus Kiel

\title{
Prof. Dr. med. Dr. rer. nat. Bernhard Kimmig zur Emeritierung
}

übernahm er als Direktor die Leitung der neu gegründeten Klinik für Strahlentherapie (Radioonkologie), die als eine von drei neuen Kliniken nach Emeritierung von Prof. Dr. Helmut Gremmel aus der ehemaligen Radiologischen Universitätsklinik hervorgegangen war.

Über 200 Beiträge und Publikationen in wissenschaftlichen Zeitschriften, Monographien und Fachbüchern umfasst sein Werk.

Neben seiner klinischen Tätigkeit übernahm er vielfältige Aufgaben in der akademischen Selbstverwaltung und im Ehrenamt. So leitete er von 1992 bis 2010 das Tumorzentrum des Klinikums, war mehr als 10 Jahre lang Mitglied des Akademischen Senats sowie der Ethikkommission und engagierte sich von 1998 bis 2008 als Vorstandsvorsitzender der SchleswigHolsteinischen Krebsgesellschaft, danach auch als Vorstandsmitglied.

Frühzeitig erkannte er die Bedeutung der bildgebenden Verfahren für die Bestrahlungsplanung. So wurde die Klinik für Strahlentherapie in Kiel eine der ersten Abteilungen, die über ein eigenes CT zur Bestrahlungsplanung verfügte.

Seiner Unterstützung ist es zu verdanken, dass im Jahre 2005 die erste universitäre Palliativstation in der Radioonkologie realisiert werden konnte, später ergänzt um eine weitere, interdisziplinäre Palliativ- und Schmerzstation als Projekt der Deutschen Krebsgesellschaft.

Wichtig ist ihm die enge Verbindung zur medizinischen Physik. Deshalb vertritt er seit Jahren die DEGRO in der Deutschen Gesellschaft für Medizinische Physik und im Normenausschuss Radiologie. Als Leiter der Ärztlichen Stelle zur
Qualitätssicherung bei der Ärztekammer Schleswig-Holstein verbindet er seine Fähigkeiten als Arzt und Physiker.

Privat gilt seine Leidenschaft antiquarischen Büchern und der Medizingeschichte. Er besitzt eine außergewöhnliche Bibliothek und eine große Zahl historischer Dokumente, so die Originalschrift Wilhelm Konrad Röntgens zur Entdeckung der Röntgenstrahlen.

Seine Mitarbeiter wünschen ihm einen aktiven Ruhestand und mehr Zeit für Freunde und die privaten Interessen.

Jürgen Schultze, Kiel, Peter Niehoff, Köln, und Rolf Sauer, Erlangen

\section{Korrespondenzadresse}

\section{Dr. J. Schultze}

Klinik für Strahlentherapie, Universitätsklinikum Schleswig-Holstein (UKSH), Campus Kiel Arnold-Heller-Str. 3, 24105 Kiel

schultze@onco.uni-kiel.de 